ESAIM: PROCEEDINGS, August 2010, Vol. 30, p. 1-14

D. Bresch, V. Calvez, E. Grenier, P. Vigneaux \& J.-F. Gerbeau, Editors

\title{
MATHEMATICAL AND NUMERICAL MODELING OF EARLY ATHEROSCLEROTIC LESIONS ${ }^{*, * *}$
}

\author{
Vincent Calvez $^{1}$, Jean Gabriel Houot ${ }^{2}$, Nicolas Meunier ${ }^{2}$, Annie Raoult ${ }^{2}$ and \\ GaBRIELA RUSNAKOVA ${ }^{3}$
}

\begin{abstract}
This article is devoted to the construction of a mathematical model describing the early formation of atherosclerotic lesions. The early stage of atherosclerosis is an inflammatory process that starts with the penetration of low density lipoproteins in the intima and with their oxidation. This phenomenon is closely linked to the local blood flow dynamics. Extending a previous work [5] that was mainly restricted to a one-dimensional setting, we couple a simple lesion growth model relying on the biomolecular process that takes place in the intima with blood flow dynamics and mass transfer. We perform numerical simulations on a two-dimensional geometry taken from $[6,7]$ that mimicks a carotid artery deformed by a perivascular cast and we compare the numerical results with experimental data.
\end{abstract}

\section{INTRODUCTION}

Atherosclerosis is an inflammatory disease that starts with intima alterations. It is now well accepted that the early stage of the process is the result of interactions between plasma low density lipoproteins that filtrate through the endothelium into the intima, cellular components (monocytes/macrophages, endothelial cells and smooth muscle cells) and the extracellular matrix of the arterial wall, see e.g. $[13,17,22]$ and references therein.

In the present work, we enrich mathematical models that were previously proposed in $[9,10]$ and $[5]$ and that were concerned with the modeling of this biomolecular process. Reference [5] tackles the modeling of the resulting atherosclerotic plaque formation. Medical evidence shows that atherosclerosis occurence is closely linked to local hemodynamic factors through the action of the shear stress exerted by the lumen blood flow on the endothelium. Therefore, relying strongly on previous works by several authors, see $[19,21,24]$, we couple the inflammatory and the lesion growth models with the blood flow by means of a mass transfer model. We are in particular interested in validating the whole model by reproducing Cheng et al. experiment $[6,7]$. This experiment that was run on mice confirms that lesions occur in preferred locations s! uch as bends and bifurcations and that their biochemical composition may depend on the location. The numerical coupling is performed in a two-dimensional setting while [5] was restricted to a one-dimensional simplified model. More

\footnotetext{
* This work was supported by the European Project ARTreat and by the Marie-Curie Actions of the European Commission in the frame of the DEASE project (MEST-CT-2005-021122).

** The authors wish to thank Julien Dambrine for helpful discussions.

${ }^{1}$ Unité de mathématiques pures et appliquées ENS Lyon, CNRS UMR 5669; e-mail: vincent.calvez@umpa.ens-lyon.fr

2 MAP5, Université Paris Descartes 45 rue des Saints Pères 75270 Paris Cedex 06. France;

e-mail: jean.houot@parisdescartes.fr; nicolas.meunier@parisdescartes.fr; Annie.Raoult@parisdescartes.fr

${ }^{3}$ Hamburg University of Technology Institute of Numerical Simulation Schwarzenbergstrasse 95, 21073 Hamburg, Germany;

e-mail: rusnakova@tu-harburg.de
}

(C) EDP Sciences, SMAI 2010 
ESAIM: PROCEEDINGS

precisely, the model is set on a domain which evolves with the disease progression: Indeed, the inflammation process modifies the shape of both the lumen and the intima. Nevertheless, we point out the fact that the intima deforms on a time scale of several months or years and that, consequently, deformation of the arterial wall under the blood flow action can be neglected.

The paper is organized as follows. We first detail the simplified inflammatory process. Then, we recall the Navier-Stokes equations that govern the blood motion in the lumen, the Darcy law that will be used to model blood filtration, as well as the equations for the evolution of LDL concentrations both in the lumen and in the intima. In so doing, we closely follow [24] and [21]. Then, we give the system of three-dimensional partial differential equations that models the inflammatory process and we propose a lesion growth model in the intima. This model relies on a matter incompressibility assumption. Sections 3 and 4 are devoted to numerical simulations in a two-dimensional domain that results from an axisymmetry hypothesis and comparisons with experimental data obtained in vivo on mice $[6,7]$.

\section{Mathematical modeling}

\subsection{Inflammatory process leading to intima deformation}

The atherosclerosis process starts with the accumulation of low density lipoproteins in the intima, where part of them are oxidized and become pathological. In order to remove the oxidized particles, circulating immune cells (e.g. monocytes) are recruited. Once in the intima, the monocytes differentiate and become macrophages that internalize the oxidized LDL. Fatty macrophages then transform into foam cells. Foam cells are responsible for the growth of a subendothelial plaque which eventually emerges in the artery lumen. In addition, the increase of macrophage concentration induces the production of pro-inflammatory cytokines, see [14,22], that contribute to recruit more monocytes.

Thanks to the previous considerations, we will consider the following simplified model:

(1) LDL penetration in the intima and LDL oxidation are the starting points for the inflammatory process.

(2) The intima LDL concentration depends on the plasma LDL concentration and on the local wall permeability.

(3) The wall permeability depends on the local hemodynamics and more precisely on the local blood shear stress acting on the arterial wall. In this respect, we assume that areas with lower wall shear stress correspond to higher penetration of LDL.

(4) When the oxidized LDL concentration exceeds a threshold, endothelial cells are activated and trigger a monocyte recruitment through the vessel wall.

(5) Plasma circulating monocytes are not in limited quantity.

(6) Incoming monocytes instantaneously differentiate into active macrophages.

(7) Active macrophages absorb oxidized LDL in the intima through a mass action law. This reaction transforms macrophages into foam cells and yields the secretion of a pro-inflammatory signal which contributes to recruit new monocytes.

(8) Newly formed foam cells are responsible for the local volume increase.

Let us now turn to equations. In Subsections 2.2 and 2.3, we make extensive use of the modeling and in-depth analysis given in $[21,24]$.

\subsection{Blood motion}

Several mathematical models were recently set up for the transport of macromolecules, such as low-density lipoproteins, from the arterial lumen to the arterial wall and inside the wall (e.g. $[15,19,21,23,24])$. The simplest model is called the wall-free model, since in this model the arterial wall is simply described by means of an appropriate boundary condition. The fluid-wall models that can be either single-layer or multilayer account for the solute dynamics not only in the lumen, but also in the arterial wall. In the present work, we consider a fluid-wall model inspired by $[21,24]$ with a single layer. This layer stands for the intima considered as a 


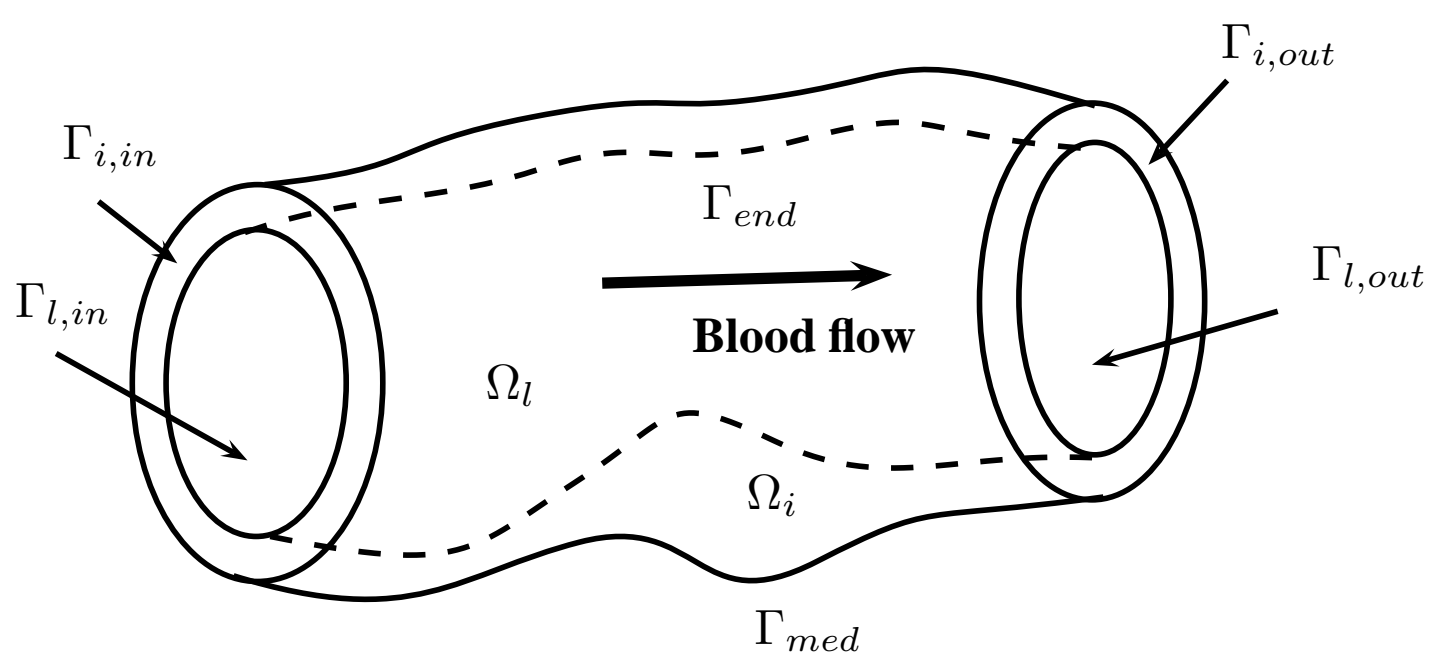

FigurE 1. Geometry

porous medium. In addition, it allows for modeling the biochemichal reactions that take place in the intima, see Subsection 2.4 .

We denote the lumen by $\Omega_{l} \subset \mathbb{R}^{3}$ and we split its boundary into the proximal section $\Gamma_{l, \text { in }}$ that corresponds to the upstream part with respect to blood flow, the distal section $\Gamma_{l, \text { out }}$ that corresponds to the downstream part with respect to blood flow, and the interface $\Gamma_{\text {end }}$ between the lumen and the arterial wall, see Figure 1. We denote the intima by $\Omega_{i} \subset \mathbb{R}^{3}$. Similarly, $\Gamma_{i, \text { in }}$ and $\Gamma_{i, \text { out }}$ correspond to the proximal and distal sections. The interface between the intima and the media is denoted by $\Gamma_{\text {med }}$. As usual, $\mathbf{n}_{l}\left(\right.$ resp. $\left.\mathbf{n}_{i}\right)$ is the outward unit normal vector to $\partial \Omega_{l}$ (resp. $\partial \Omega_{i}$ ).

Let us now focus on the various quantities that will be involved in the biomechanical part of the model which consists of fluid dynamics and mass transfer equations. In what follows, for $\mathbf{x} \in \Omega_{l}$ and $t>0, \mathbf{u}_{l}(t, \mathbf{x}) \in \mathbb{R}^{3}$ will denote the blood velocity field in the lumen and $p_{l}(t, \mathbf{x})$ the pressure. Moreover, $c_{l}(t, \mathbf{x})$ will denote the plasma LDL concentration. Similarly, for $\mathbf{x} \in \Omega_{i}$ and $t>0, \mathbf{u}_{i}(t, \mathbf{x}) \in \mathbb{R}^{3}$ will denote the blood velocity field in the intima, $p_{i}(t, \mathbf{x})$ the pressure, and $c_{i}(t, \mathbf{x})$ the intima native (not oxidized) LDL concentration.

\subsubsection{In the lumen}

We make the following assumptions:

(1) Blood is an incompressible Newtonian fluid with density $\rho$ and dynamic viscosity $\nu$.

(2) Deformation of the arterial wall under the blood flow action can be neglected.

Remark 2.1. The first assumption is reasonable since atherosclerosis usually concerns medium or large arteries. As for the second assumption, our primary interest here is the rise of an atheromatous plaque which obviously deforms the arterial wall. Since the time scale of the plaque formation is much larger (months or years) than the time scale of the blood flow (less than 1s), we may ignore the pulsatile nature of blood flow. 
Under the previous assumptions, the blood motion in the lumen can be mathematically described by means of the classical Navier-Stokes equations:

$$
\begin{aligned}
\rho\left[\partial_{t} \mathbf{u}_{l}+\left(\mathbf{u}_{l} \cdot \nabla\right) \mathbf{u}_{l}\right]-\nu \Delta \mathbf{u}_{l}+\nabla p_{l} & =0, & & \mathbf{x} \in \Omega_{l}, t \in[0, T], \\
\nabla \cdot \mathbf{u}_{l} & =0, & & \mathbf{x} \in \Omega_{l}, t \in[0, T], \\
\mathbf{u}_{l} & =\mathbf{U}_{l, \text { in }}, & & \mathbf{x} \text { on } \Gamma_{l, \text { in }}, t \in[0, T], \\
\mathbf{T}\left(\mathbf{u}_{l}, p_{l}\right) \mathbf{n}_{l} & =-p_{\text {out }} \mathbf{n}_{l}, & & \mathbf{x} \text { on } \Gamma_{l, \text { out }}, t \in[0, T], \\
\mathbf{u}_{l} \cdot \mathbf{n}_{l} & =J_{v}, & & \mathbf{x} \text { on } \Gamma_{\text {end }}, t \in[0, T], \\
\mathbf{u}_{l}-\left(\mathbf{u}_{l} \cdot \mathbf{n}_{l}\right) \mathbf{n}_{l} & =0, & & \mathbf{x} \text { on } \Gamma_{\text {end }}, t \in[0, T],
\end{aligned}
$$

where

$$
\mathbf{T}(\mathbf{u}, p):=2 \nu \mathbf{D}(\mathbf{u})-p \mathrm{Id},
$$

is the Cauchy stress tensor and $\mathbf{D}(\mathbf{u}):=\frac{1}{2}\left(\nabla \mathbf{u}+\nabla \mathbf{u}^{T}\right)$ is the rate of deformation tensor. At the inlet $\Gamma_{l, i n}$, the entering velocities are given by a Poiseuille profile $\mathbf{U}_{l, i n}$. Such a Dirichlet boundary condition is generally acceptable provided the inflow section is sufficiently upstream the site of interest. The Neumann boundary condition at the artificial outlet means that the force applied on the fluid contained in $\Omega_{l}$ by the fluid outside of $\Omega_{l}$ is supposed to be close to a pressure load. Finally, we suppose the arterial wall $\Gamma_{\text {end }}$ to be permeable to blood. Indeed, equation (2.1e) means that there exists a blood flux. This flux will obey Kedem-Katchalsky equations, see (2.7a) that give a law for $J_{v}$. Boundary condition (2.1f) states that the blood velocity tangential component is null, this is a standard no-slip boundary condition.

\subsubsection{In the intima}

Following again $[21,24]$, we describe the blood filtration in the wall by the Darcy model:

$$
\begin{array}{rlrl}
\mathbf{u}_{i} & =-\frac{K}{\mu} \nabla p_{i}, & \mathbf{x} \in \Omega_{i}, t \in[0, T], \\
\nabla \cdot \mathbf{u}_{i} & =0, & & \mathbf{x} \in \Omega_{i}, t \in[0, T], \\
\mathbf{u}_{i} \cdot \mathbf{n}_{i} & =0, & & \mathbf{x} \text { on } \Gamma_{i, i n} \cup \Gamma_{i, \text { out }}, t \in[0, T], \\
\mathbf{u}_{i} \cdot \mathbf{n}_{i} & =-J_{v}, & & \mathbf{x} \text { on } \Gamma_{\text {end }}, t \in[0, T], \\
p_{i} & =p_{\text {med }} & & \mathbf{x} \text { on } \Gamma_{\text {med }}, t \in[0, T],
\end{array}
$$

where $K$ is the permeability of $\Omega_{i}, \mu$ its viscosity, and $p_{m e d}$ is a given pressure on the media interface. Condition (2.3d) expresses the continuity of normal blood velocities across the endothelium.

\subsection{LDL evolution}

\subsubsection{In the lumen}

Classically, the evolution of the plasma LDL concentration is given by a convection-diffusion equation

$$
\begin{aligned}
\partial_{t} c_{l}+\nabla \cdot\left(-D_{l} \nabla c_{l}+\mathbf{u}_{l} c_{l}\right) & =0, & & \mathbf{x} \in \Omega_{l}, t \in[0, T], \\
c_{l} & =C_{l, \text { in }}, & & \mathbf{x} \text { on } \Gamma_{l, \text { in }}, t \in[0, T], \\
\nabla c_{l} \cdot \mathbf{n}_{l} & =0, & & \mathbf{x} \text { on } \Gamma_{l, \text { out }}, t \in[0, T], \\
\left(-D_{l} \nabla c_{l}+\mathbf{u}_{l} c_{l}\right) \cdot \mathbf{n}_{l} & =J_{s}, & & \mathbf{x} \text { on } \Gamma_{\text {end }}, t \in[0, T],
\end{aligned}
$$

where $D_{l}$ is the diffusion coefficient of the LDL in the lumen, $C_{l, i n}$ is assumed to be known. Condition (2.4d) states that the total LDL flux on $\Gamma_{\text {end }}$ is given by $J_{s}$ whose form will be defined by Kedem-Katchalsky equations, see $(2.7 \mathrm{a})-(2.7 \mathrm{~b})$. 


\subsubsection{In the intima}

By considering a fluid-wall model, we allow for a discontinuous LDL concentration across the endothelium. More precisely, the native LDL concentration $c_{i}$ in the intima is not a given datum and its dynamics is governed by a convection-diffusion equation that is similar to $(2.4 \mathrm{a})-(2.4 \mathrm{~d})$ except for the presence of a degradation term accounting for LDL oxidation in the intima with rate $r_{o x}$. Oxidation is expected to be a rare event and $r_{o x}$ is small. Disregarding the fact that in order to get oxidized low density lipoproteins should stay long enough in the intima, we use a standard immediate exponential decay model.

Therefore, the evolution equation of intima native LDL concentration reads

$$
\begin{aligned}
\partial_{t} c_{i}+\nabla \cdot\left(-D_{i} \nabla c_{i}+\mathbf{u}_{i} c_{i}\right) & =-r_{o x} c_{i}, & & \mathbf{x} \in \Omega_{i}, t \in[0, T], \\
\nabla c_{i} \cdot \mathbf{n}_{i} & =0, & & \mathbf{x} \text { on } \Gamma_{i, \text { in }} \cup \Gamma_{i, \text { out }} \cup \Gamma_{\text {med }}, t \in[0, T], \\
\left(-D_{i} \nabla c_{i}+\mathbf{u}_{i} c_{i}\right) \cdot \mathbf{n}_{i} & =-J_{s}, & & \mathbf{x} \text { on } \Gamma_{\text {end }}, t \in[0, T],
\end{aligned}
$$

where $D_{i}$ is the LDL diffusion coefficient in the intima.

\subsubsection{Kedem-Katchalsky equations}

Kedem-Katchalsky equations prescribe the fluxes of solvent and solute through a semi-permeable membrane that separates two solutions where solute concentrations and fluid pressures are nonequal, see [11], [12]. Letting $\delta p=p_{l}-p_{i}$ and $\delta c=c_{l}-c_{i}$, they read under general form

$$
\begin{aligned}
& J_{v}=L_{p}(\delta p-\alpha \delta c) \\
& J_{s}=\xi \delta c+\beta J_{v}
\end{aligned}
$$

where $L_{p}$ is the hydraulic conductivity and $\xi$ is the local membrane permeability that may depend on the local flow. Equations (2.6a)-(2.6b) clearly induce a strong coupling between the blood model and the LDL model. Indeed the condition on the blood flux makes use of the concentration drop. Conversely, the condition on the LDL flux makes use of the blood pressure drop and possibly of other features of the blood flow by means of $\xi$. In the present work, we drastically simplify Kedem-Katchalsky equations by assuming that

$$
\begin{aligned}
& J_{v}=L_{p}\left(p_{l}-p_{i}\right), \\
& J_{s}=\xi\left(c_{l}-c_{i}\right) .
\end{aligned}
$$

As a consequence, the blood model that consists of Navier-Stokes equations (2.1) and Darcy equations (2.3) can be solved for itself. Then, the blood velocities are an input in the left-hand sides of systems (2.4) and (2.5) that govern native LDL concentrations. The right-hand sides of (2.4) and (2.5) also call to the blood model in the following way. Medical evidence shows that regions with shear stress of low magnitude and regions with oscillatory shear stress are more likely to develop atheromatous plaques while high shear stress regions appear to be protected from atherosclerosis. We recall that the shear stress exerted by the blood flow on the arterial wall is given by $-\boldsymbol{\sigma}\left(\mathbf{u}_{l}, p_{l}\right)$ where

$$
\boldsymbol{\sigma}\left(\mathbf{u}_{l}, p_{l}\right)=\mathbf{T}\left(\mathbf{u}_{l}, p_{l}\right) \mathbf{n}_{l}-\left(\mathbf{n}_{l}^{T} \mathbf{T}\left(\mathbf{u}_{l}, p_{l}\right) \mathbf{n}_{l}\right) \mathbf{n}_{l}
$$

We incorporate this phenomenon in the model by choosing a shear stress-permeability relationship

$$
\xi(\boldsymbol{\sigma})=a_{0} \log \left(1+\frac{a_{1}}{|\boldsymbol{\sigma}|+a_{2}}\right)
$$

similar to the law proposed in [24] for the oxygen. Coefficients $a_{0}, a_{1}$ and $a_{2}$ are positive and will be made precise later on. Such a permeability is 
(1) a positive function in order to enforce the LDL flux to be directed towards the intima when the LDL drop is positive,

(2) a decreasing function of $|\boldsymbol{\sigma}|$ thus assigning higher permeability to areas with low shear stress.

\subsection{Inflammatory process}

Let us now turn to the modeling of the biochemical process. As mentioned earlier, this is a slow process that gives rise to an atheromatous plaque over a period of months or years: the growth velocity that will be obtained below is expected to be quite small. For all $x \in \Omega_{i}$ and for all $t>0$, we respectively denote by $c_{o x}(t, \mathbf{x}), M(t, \mathbf{x})$, $S(t, \mathbf{x}), F(t, \mathbf{x})$ the concentrations of oxidized LDL, macrophages, cytokines, and foam cells in the intima. In addition, $W(t, \mathbf{x})$ stands for the biomass which includes smooth muscle cells, the extracellular matrix and the interstitial fluid.

\subsubsection{Foam cells, biomass and plaque growth}

The basic idea for the plaque growth model is that, by absorbing oxidized LDL, macrophages transform into foam cells that are large cells. By restricting to foam cells and biomass, since other constituents are much smaller, the incompressibility assumption used by many authors [1], [4], [2], [3] in tumor growth modeling reads

$$
F+W=A,
$$

where $A$ denotes the total number of cells per unit volume assumed to be invariant along time and space. Foam cell formation induces rearrangement and global domain deformation with velocity $\mathbf{v}$. The foam cell concentration evolution is given by

$$
\partial_{t} F+\operatorname{div}(\mathbf{v} F)=k_{F} c_{o x} \cdot M, \mathbf{x} \in \Omega_{i}, t \in[0, T] .
$$

This is a transport equation for $F$ with a creation term coming from the absorption of oxidized LDL by macrophages: In the present modeling, a simple mass action law $M+c_{o x} \rightarrow F$ has been chosen. The second left-hand side term states that foam cells are transported with velocity $\mathbf{v}$. Note that because of their large size foam cells do not diffuse.

Under our assumptions, smooth muscle cells and fibers do not contribute to the inflammatory process. Therefore, $W$ satisfies a simple transport equation with no reaction term

$$
\partial_{t} W+\operatorname{div}(\mathbf{v} W)=0, \mathbf{x} \in \Omega_{i}, t \in[0, T] .
$$

By simply adding equations (2.10) and (2.11), we obtain a first information on v. Indeed,

$$
\partial_{t}(F+W)+\operatorname{div}(\mathbf{v}(F+W))=k_{F} c_{o x} \cdot M
$$

which yields

$$
\operatorname{div} \mathbf{v}=\frac{k_{F}}{A} c_{o x} \cdot M
$$

Equation (2.12) is not sufficient to determine $\mathbf{v}$ and in order to close the system we need an additional equation. We choose to use a simple phenomenological model inspired by the Stokes equation; for a discussion of such models see [1], [4], [2], [3]. The system reads

$$
\begin{aligned}
-\operatorname{div} \mathbf{D}(\mathbf{v})+\nabla \mathrm{q} & =0, & & \mathbf{x} \in \Omega_{i}, t \in[0, T], \\
\operatorname{div} \mathbf{v} & =\frac{k_{F}}{A} c_{o x} \cdot M, & & \mathbf{x} \in \Omega_{i}, t \in[0, T], \\
\mathbf{D}(\mathbf{v}) \mathbf{n}_{i}-q \mathbf{n}_{i} & =0, & & \mathbf{x} \text { on } \Gamma_{\text {end }}, t \in[0, T], \\
\mathbf{v} & =0, & & \mathbf{x} \text { on } \partial \Omega_{i} \backslash \Gamma_{\text {end }}, t \in[0, T] .
\end{aligned}
$$


Note that (2.13d) implicitly assumes that smooth muscle cells and other species have zero velocity at the media boundary, an hypothesis that should be removed in further work.

As expected, the divergence of the velocity field is nonnegative. This creates a movement of the cells from the center of the domain to the boundary and induces an increase of the tissue volume. As a consequence, as already stated, domains $\Omega_{l}$ and $\Omega_{i}$ depend on time and the interface $\Gamma_{e n d}$ deforms with velocity $\mathbf{v}$. Determining $\mathbf{v}$ requires knowledge of $c_{o x}$ and $M$; this is the object of the next subsections.

\subsubsection{Oxidized $L D L$}

Oxidized LDL, macrophages and cytokines are of small size with respect to foam cells. Therefore, we assume that they undergo Brownian motion. Although the diffusion coefficients $d_{o x}, d_{M}$ and $d_{S}$ are small because of the intima structure, the growth process is so slow that we can ignore transport terms for these species. Altogether, the system modeling the evolution of the oxidized LDL concentration $c_{o x}$ in the intima reads

$$
\begin{array}{lr}
\partial_{t} c_{o x}=d_{o x} \Delta c_{o x}-k_{F} c_{o x} \cdot M+r_{o x} c_{i}, & \mathbf{x} \in \Omega_{i}, t \in[0, T], \\
\partial_{\mathbf{n}_{i}} c_{o x}=0, & \mathbf{x} \text { on } \partial \Omega_{i}, t \in[0, T],
\end{array}
$$

where $k_{F}$ has been introduced in (2.10) and $r_{o x}$ in (2.5a).

Remark 2.2. ¿From the Kedem-Katchalsky model, the concentration $c_{i}$ of native LDL in the intima depends on the permeability $\xi$ of the endothelial membrane, which in turn has been supposed to depend on the wall shear stress. As a consequence, the final term in (2.14a) couples the inflammatory model in the arterial wall with the blood flow. This is the main novelty of this work.

\subsubsection{Macrophage}

The system that models the evolution of macrophage density is

$$
\begin{aligned}
& \partial_{t} M=d_{M} \Delta M-k_{F} c_{o x} \cdot M, \\
& \partial_{\mathbf{n}_{i}} M=-f(S), \\
& \partial_{\mathbf{n}_{i}} M=0,
\end{aligned}
$$

$$
\begin{array}{r}
\mathbf{x} \in \Omega_{i}, t \in[0, T], \\
\mathbf{x} \text { on } \Gamma_{\text {end }}, t \in[0, T], \\
\mathbf{x} \text { on } \partial \Omega_{i} \backslash \Gamma_{\text {end }}, t \in[0, T],
\end{array}
$$

with a diffusion coefficient $d_{M}$ even smaller than $d_{o x}$. The boundary condition on the endothelium is the main point of the model and states that the macrophage recruitment is due to a pro-inflammatory signal $S$. In this signal we include both chemokines and cytokines. Physically speaking, wall adhesion properties are modified and circulating immune cells are recruited via adhesion on the endothelium and invagination. The response function $f$ has to be positive in order to allow macrophages to penetrate into the intima and to prevent them to leave. In addition, $f$ is an increasing function with a saturation effect due to the limited transfer rate of monocytes across the vessel wall. We simply choose

$$
f(S)=\frac{S}{1+S} .
$$

\subsubsection{Signal}

Finally, the chemoattractant is given by

$$
\begin{array}{lr}
\partial_{t} S=d_{S} \Delta S-\lambda S+\gamma\left(c_{o x}-c_{o x}^{t h}\right)_{+}+k_{F} c_{o x} \cdot M, & \mathbf{x} \in \Omega_{i}, t \in[0, T], \\
\partial_{\mathbf{n}_{i}} S=0, & \mathbf{x} \text { on } \partial \Omega_{i}, t \in[0, T] .
\end{array}
$$

The third term in the right-hand side of (2.16a) expresses that the chemoattractant is created when the oxidized LDL concentration exceeds a threshold. The fourth term expresses that it is created as well by the reaction $M+C_{O x} \rightarrow F$. Finally, the signal undergoes degradation with rate $\lambda$. 


\section{Numerical PROCEDURE}

Our aim is to make visible the intima deformation resulting from the reactions that take place in the arterial wall and to compute several quantities of interest. The general organization of the iterative numerical procedure with time step $\Delta t>0$ is as follows. We start from an initial shape and from initial values for the hydrodynamic and biochemical quantities. Then, assuming that approximate domains $\Omega_{l}^{n-1}, \Omega_{i}^{n-1}$ at time $t_{n-1}=(n-1) \Delta t$, approximate values $\mathbf{u}_{l}^{n-1}, p_{l}^{n-1}, c_{l}^{n-1}$ defined on $\Omega_{l}^{n-1}$, approximate values $\mathbf{u}_{i}^{n-1}, p_{i}^{n-1}, c_{i}^{n-1}, c_{o x}^{n-1}, M^{n-1}$, $S^{n-1}$ defined on $\Omega_{i}^{n-1}$ and an overall growth velocity $\mathbf{v}^{n-1}$ are known, computations that lead to approximate values at time $t_{n}=n \Delta t$ split into three parts:

(1) First, we solve numerically at time $t_{n}$ on $\Omega_{l}^{n-1} \cup \Omega_{i}^{n-1}$ the equations of the sole space variable that govern blood filtration and native LDL transfer. In particular, we compute the native LDL concentration in the intima. The approximate values at this stage are still defined on $\Omega_{l}^{n-1}$ or $\Omega_{i}^{n-1}$ and are denoted by $\hat{\mathbf{u}}_{l}^{n}, \hat{p}_{l}^{n}, \hat{c}_{l}^{n}$ and by $\hat{\mathbf{u}}_{i}^{n}, \hat{p}_{i}^{n}, \hat{c}_{i}^{n}$.

(2) Second, we solve on $\Omega_{i}^{n-1}$ the equations that govern the inflammatory process with the intima native LDL concentration obtained above as an input. This provides $\hat{c}_{o x}^{n}, \hat{M}^{n}, \hat{S}^{n}$ defined on $\Omega_{i}^{n-1}$.

(3) Third, we compute the plaque growth velocity $\hat{\mathbf{v}}^{n}$ defined on $\Omega_{i}^{n-1}$. We extend it to $\Omega_{l}^{n-1}$ by a standard trick, and we move $\Omega_{i}^{n-1}$ and $\Omega_{l}^{n-1}$ accordingly. This provides the updated domains $\Omega_{i}^{n}$ and $\Omega_{l}^{n}$. Finally, we transport all unknowns computed in parts 1 and 2, as well as the growth velocity, on these updated domains and we denote them by $\mathbf{u}_{l}^{n}, p_{l}^{n}, c_{l}^{n}, \mathbf{u}_{i}^{n}, p_{i}^{n}, c_{i}^{n}, c_{o x}^{n}, M^{n}, S^{n}, \mathbf{v}^{n}$.

Let us now describe more precisely the numerical schemes we use for each of the above steps.

\subsection{Hemodynamics and native intima LDL transfer}

The loop leading to values at time $t_{n-1}$ is supposed to be completed. Therefore, domains $\Omega_{l}^{n-1}$ and $\Omega_{i}^{n-1}$, functions $\mathbf{u}_{l}^{n-1}, \mathbf{u}_{i}^{n-1}, p_{l}^{n-1}, p_{i}^{n-1}, c_{l}^{n-1}, c_{i}^{n-1}, \mathbf{v}^{n-1}$ are given. These are the data we use to obtain the approximate values $\hat{\mathbf{u}}_{l}^{n}, \hat{p}_{l}^{n}, \hat{c}_{l}^{n}$ on $\Omega_{l}^{n-1}$ and $\hat{\mathbf{u}}_{i}^{n}, \hat{p}_{i}^{n}, \hat{c}_{i}^{n}$ on $\Omega_{i}^{n-1}$ :

- We solve at time $t_{n}$ on $\Omega_{l}^{n-1}$ the static numerical counterpart of the system consisting of the NavierStokes system (2.1) and of the Darcy model (2.3). Recall that because of the simplified KedemKatchalsky equation (2.7a) we chose, equations (2.1)-(2.3)-(2.7a) make a closed system with unknowns $\mathbf{u}_{l}, p_{l}, \mathbf{u}_{i}, p_{i}$. At the end of this step, $\hat{\mathbf{u}}_{l}^{n}, \hat{p}_{l}^{n}, \hat{\mathbf{u}}_{i}^{n}, \hat{p}_{i}^{n}$ are determined.

- We solve at time $t_{n}$ on $\Omega_{l}^{n-1}$ the static numerical counterpart of the LDL model, namely the system consisting of $(2.4),(2.5)$ with the boundary data $J_{s}$ obeying $(2.7 \mathrm{~b})$. At the end of this step, concentrations $\hat{c}_{l}^{n}$ and $\hat{c}_{i}^{n}$ are determined.

Two points have to be made precise: First, the method we use to approximate time derivatives since this is the way the systems above that are independent of $t$ are set. Second, how we deal with the fact that each system is posed on two separate domains.

Time derivatives and $A L E$ correction. Let us concentrate on the simple case of a linear transport equation similar to (2.4a) or to (2.5a) except for the fact that the space domain $\Omega \subset \mathbb{R}^{d}$ does not evolve with time: given a transport velocity $\mathbf{w}:[0, T] \times \Omega \mapsto \mathbb{R}^{d}$ and a function $g:[0, T] \times \mathbb{R}^{d} \mapsto \mathbb{R}$, find $c:[0, T] \times \Omega \mapsto \mathbb{R}$ such that

$$
\partial_{t} c+\nabla \cdot(\mathbf{w} c)-\Delta c=g .
$$

Assume that $\operatorname{div} \mathbf{w}=0$ which will be true in the cases under consideration. Then (3.1) reads

$$
\partial_{t} c+\mathbf{w} \cdot \nabla c-\Delta c=g .
$$

For every $\mathbf{x}$ in $\Omega$, the characteristic curve passing through $\left(t_{n}, \mathbf{x}\right)$ is the solution of the ordinary differential system in $\mathbb{R}^{d}$

$$
\forall t \in[0, T], \quad \dot{\mathbf{X}}(t ; \mathbf{x}, \mathbf{w})=\mathbf{w}(t, \mathbf{X}(t ; \mathbf{x}, \mathbf{w})), \quad \mathbf{X}\left(t_{n} ; \mathbf{x}, \mathbf{w}\right)=\mathbf{x} .
$$


Relying on the identity $\left(\partial_{t} c+\mathbf{w} \cdot \nabla c\right)(t, \mathbf{x})=C^{\prime}(t)$ where $C(t)=c(t, \mathbf{X}(t ; \mathbf{x}, \mathbf{w}))$, the method of characteristics chooses the following approximation formula

$$
\left(\partial_{t} c+\mathbf{w} \cdot \nabla c\right)\left(t_{n}, \mathbf{x}\right) \approx \frac{C\left(t_{n}\right)-C\left(t_{n-1}\right)}{\Delta t}=\frac{c\left(t_{n}, \mathbf{x}\right)-c\left(t_{n-1}, \mathbf{X}\left(t_{n-1} ; \mathbf{x}, \mathbf{w}\right)\right)}{\Delta t} .
$$

Therefore, approximate functions $c^{n}$ at time $t_{n}$ are generated by solving the static problem

$$
\frac{c^{n}(\cdot)-c^{n-1}\left(\mathbf{X}\left(t_{n-1} ; \cdot, \mathbf{w}\right)\right)}{\Delta t}-\Delta c^{n}(\cdot)=g\left(t_{n}, \cdot\right),
$$

or equivalently, letting $\chi=(\Delta t)^{-1}$,

$$
\chi c^{n}(\cdot)-\Delta c^{n}(\cdot)=\chi c^{n-1}\left(\mathbf{X}\left(t_{n-1} ; \cdot, \mathbf{w}\right)\right)+g\left(t_{n}, \cdot\right) .
$$

Let us now turn to the more complex case when $\Omega$ in equation (3.1) is no longer a given domain but evolves with time. Such is the case for systems (2.4) and (2.5). Suppose that the numerical scheme has already provided the approximate domain $\Omega^{n-1}$ at time $t_{n-1}$ and the deformation velocity $\mathbf{v}^{n-1}$ of $\Omega^{n-1}$, and suppose that an approximate value $\mathbf{w}^{n-1}$ of $\mathbf{w}\left(t_{n-1}\right)$ on $\Omega^{n-1}$ is available. For a given field $\mathbf{z}$ on $\Omega^{n-1}$, let now $\mathbf{X}$ be the solution of

$$
\forall t \in\left[t_{n-1}, t_{n}\right], \forall \mathbf{x} \in \Omega^{n-1}, \quad \dot{\mathbf{X}}(t ; \mathbf{x}, \mathbf{z})=\mathbf{z}(\mathbf{X}(t ; \mathbf{x}, \mathbf{z})), \quad \mathbf{X}\left(t_{n} ; \mathbf{x}, \mathbf{z}\right)=\mathbf{x} .
$$

Then, the ALE correction, see $[8,16]$, to the method of characteristics consists in first generating a function $\hat{c}^{n}$ defined on $\Omega^{n-1}$ by

$$
\frac{\hat{c}^{n}(\cdot)-c^{n-1}\left(\mathbf{X}\left(t_{n-1} ; \cdot, \mathbf{w}^{n-1}-\mathbf{v}^{n-1}\right)\right.}{\Delta t}-\Delta \hat{c}^{n}(\cdot)=g\left(t_{n}, \cdot\right),
$$

or, equivalently,

$$
\chi \hat{c}^{n}(\cdot)-\Delta \hat{c}^{n}(\cdot)=\chi c^{n-1}\left(\mathbf{X}\left(t_{n-1} ; \cdot, \mathbf{w}^{n-1}-\mathbf{v}^{n-1}\right)+g\left(t_{n}, \cdot\right),\right.
$$

then in updating $\hat{c}^{n}$ in $c^{n}$ on $\Omega^{n}$.

The time discretization of the Navier-Stokes system (2.1) follows the same lines with the additional ingredient of freezing the first velocity in the nonlinear term $\left(\mathbf{u}_{l} \cdot \nabla\right) \mathbf{u}_{l}$ to its value at time $t_{n-1}$ while generating values at time $t_{n}$. Let us emphasize the fact that for all computations we used the finite element solver FreeFem++ where the algorithms we just described are implemented.

Domain decomposition. Once the time discretization is performed, we have to solve static problems that lead to $\left(\hat{\mathbf{u}}_{l}^{n}, \hat{\mathbf{u}}_{i}^{n}, \hat{p}_{l}^{n}, \hat{p}_{i}^{n}\right)$ on the one hand and to $\left(\hat{c}_{l}^{n}, \hat{c}_{i}^{n}\right)$ on the other hand. In each system some unknowns are defined on $\Omega_{l}^{n-1}$, others are defined on $\Omega_{i}^{n-1}$, and equations on the separate domains are coupled through the interface conditions (2.1e)-(2.3d) that call to (2.7a) for the first system and through the interface conditions (2.1e)-(2.3d) that call to $(2.7 \mathrm{~b})$ for the second system. Solving these systems is done by means of a classical domain decomposition method, namely a Richardson method, see [20] and particularly [24] for the present situation.

Let us describe the way $\left(\hat{\mathbf{u}}_{l}^{n}, \hat{\mathbf{u}}_{i}^{n}, \hat{p}_{l}^{n}, \hat{p}_{i}^{n}\right)$ is obtained. The procedure consists in constructing a sequence $\left(\mathbf{P}^{k}\right)_{k \geq 0}=\left(\hat{\mathbf{u}}_{l}^{k, n-1}, \hat{p}_{l}^{k, n-1}, \hat{p}_{i}^{k, n-1}\right)_{k \geq 0}$ with $\mathbf{P}^{0}=\left(\mathbf{u}_{l}^{n-1}, p_{l}^{n-1}, p_{i}^{n-1}\right)$ by means of 
- a system whose the input relative to the $k$-loop is $\left(\hat{p}_{l}^{k-1, n-1}, \hat{p}_{i}^{k-1, n-1}\right)$ and output is $\left(\hat{\mathbf{u}}_{l}^{k, n-1}, \hat{p}_{l}^{k, n-1}\right)$ :

$$
\begin{aligned}
\rho \chi \hat{\mathbf{u}}_{l}^{k, n-1}-\nu \Delta \hat{\mathbf{u}}_{l}^{k, n-1}+\nabla \hat{p}_{l}^{k, n-1} & =\rho \chi \mathbf{u}_{l}^{n-1}\left(\mathbf{X}\left(t_{n-1} ; \cdot, \mathbf{u}_{l}^{n-1}-\mathbf{v}^{n-1}\right),\right. & & \mathbf{x} \in \Omega_{l}^{n-1}, \\
\nabla \cdot \hat{\mathbf{u}}_{l}^{k, n-1} & =0, & & \mathbf{x} \in \Omega_{l}^{n-1}, \\
\hat{\mathbf{u}}_{l}^{k, n-1} & =\mathbf{U}_{l, \text { in }}, & & \mathbf{x} \text { on } \Gamma_{l, \text { in }}^{n-1}, \\
\mathbf{T}\left(\hat{\mathbf{u}}_{l}^{k, n-1}, \hat{p}_{l}^{k, n-1}\right) \mathbf{n}_{l} & =-p_{\text {out }} \mathbf{n}_{l}, & & \mathbf{x} \text { on } \Gamma_{l, \text { out }}^{n-1}, \\
\hat{\mathbf{u}}_{l}^{k, n-1} \cdot \mathbf{n}_{l} & =L_{p}\left(\hat{p}_{l}^{k-1, n-1}-\hat{p}_{i}^{k-1, n-1}\right), & & \mathbf{x} \text { on } \Gamma_{\text {end }}^{n-1}, \\
\hat{\mathbf{u}}_{l}^{k, n-1}-\left(\hat{\mathbf{u}}_{l}^{k, n-1} \cdot \mathbf{n}_{l}\right) \mathbf{n}_{l} & =0, & & \mathbf{x} \text { on } \Gamma_{\text {end }}^{n-1},
\end{aligned}
$$

- a system whose the input relative to the $k$-loop is $\left(\hat{p}_{l}^{k, n-1}, \hat{p}_{i}^{k-1, n-1}\right)$ and output is $\hat{p}_{i}^{k, n-1}$, or equivalently $\left(\hat{\mathbf{u}}_{i}^{k, n-1}, \hat{p}_{i}^{k, n-1}\right)$ :

$$
\begin{array}{rlr}
\hat{\mathbf{u}}_{i}^{k, n-1} & =-\frac{K}{\mu} \nabla \hat{p}_{i}^{k, n-1}, & \mathbf{x} \in \Omega_{i}^{n-1}, \\
\nabla \cdot \hat{\mathbf{u}}_{i}^{k, n-1} & =0, & \mathbf{x} \in \Omega_{i}^{n-1}, \\
\hat{\mathbf{u}}_{i}^{k, n-1} \cdot \mathbf{n}_{i} & =0, & \mathbf{x} \text { on } \Gamma_{i, i n}^{n-1} \cup \Gamma_{i, \text { out }}^{n-1}, \\
\hat{\mathbf{u}}_{i}^{k, n-1} \cdot \mathbf{n}_{i} & =-L_{p}\left(\hat{p}_{l}^{k, n-1}-\hat{p}_{i}^{k-1, n-1}\right), & \mathbf{x} \text { on } \Gamma_{\text {end }}^{n-1}, \\
\hat{p}_{i}^{k, n-1} & =p_{\text {med }} & \mathbf{x} \text { on } \Gamma_{\text {med }}^{n-1} .
\end{array}
$$

Note that in equation (3.9a) the right-hand side term is computed once and for all from the values available at the start of the $k$-loops. Iterations are stopped when a standard criterion $\left\|\mathbf{P}^{k}-\mathbf{P}^{k-1}\right\|_{2} \leq \varepsilon\left\|\mathbf{P}^{k}\right\|_{2}$ is fulfilled where $\|\cdot\|_{2}$ is the $L^{2}$ norm. Because of the smallness of $L_{p}$, the coupling is weak and convergence in $k$ is fast: for $\varepsilon=10^{-12}$ the process can be stopped after five iterations. Current quantities at the iterative procedure stop provide the values $\hat{\mathbf{u}}_{l}^{n}, \hat{p}_{l}^{n}, \hat{\mathbf{u}}_{i}^{n}, \hat{p}_{i}^{n}$.

A similar method is used for the derivation of $\left(\hat{c}_{l}^{n}, \hat{c}_{i}^{n}\right)$ from $\left(c_{l}^{n-1}, c_{i}^{n-1}\right)$. In all loops in $k$, the convection velocity is taken to its value at the start of the step $n-1$ to $n$, namely $\mathbf{u}_{l}^{n-1}$, thus making the total numerical velocity with ALE correction be $\mathbf{u}_{l}^{n-1}-\mathbf{v}^{n-1}$ as above. As an input for $J_{s}$, we need an approximate permeability. We take it from the values available at the start of the loops as well, and we define $\xi^{n-1}=\xi\left(\overline{\boldsymbol{\sigma}}^{n-1}\right)$, see $(2.9)$, where $\overline{\boldsymbol{\sigma}}^{n-1}$ is an approximate value of the wall shear stress $\boldsymbol{\sigma}^{n-1}:=\boldsymbol{\sigma}\left(\mathbf{u}_{l}^{n-1}, p_{l}^{n-1}\right)$, see (2.8). As the coupling plays a real role here, convergence is slower than for the hydrodynamic part. Indeed, for a similar criterion, 20 to 30 iterations are necessary for $\varepsilon=10^{-12}$. Nevertheless, we $\mathrm{f}$ ! ound the convergence rate acceptable and we did not use the relaxation method proposed in [24]. Current quantities at the iterative procedure stop provide $\hat{c}_{l}^{n}, \hat{c}_{i}^{n}$.

\subsection{Inflammatory part}

Recall that the inflammatory model consists of the reaction-diffusion equations (2.14), (2.15) and (2.16) and that it is coupled to the LDL transfer model by the sole term $c_{i}$. The value $\hat{c}_{i}^{n}$ that has just been obtained is taken as an input for this coupling term in the present step $n-1$ to $n$. The system is solved on $\Omega_{i}^{n-1}$ by means of a standard implicit method with ALE convection. More precisely, the discrete versions of equations (2.14), (2.15) and (2.16) are decoupled by computing first $\hat{c}_{o x}^{n}$ by means of $\left(\hat{c}_{o x}^{n-1}, \hat{M}^{n-1}\right)$, then $\hat{M}^{n}$ by means of $\left(\hat{c}_{o x}^{n}\right.$, $\left.\hat{M}^{n-1}, \hat{S}^{n-1}\right)$, and finally $\hat{S}^{n}$ by means of $\left(\hat{c}_{o x}^{n}, \hat{M}^{n}, \hat{S}^{n-1}\right)$. The procedure reads 
FigURE 2. Geometry of the lumen at rest

$$
\begin{aligned}
\chi \hat{c}_{o x}^{n}-d_{o x} \Delta \hat{c}_{o x}^{n}+k_{F} \hat{c}_{o x}^{n} \cdot \hat{M}^{n-1} & =r_{o x} \hat{c}_{i}^{n}+\chi c_{o x}^{n-1} \circ\left(-\mathbf{v}^{n-1}\right), \\
\chi \hat{M}^{n}-d_{M} \Delta \hat{M}^{n}+k_{F} \hat{c}_{o x}^{n} \cdot \hat{M}^{n} & =\chi M^{n-1} \circ\left(-\mathbf{v}^{n-1}\right), \quad \text { with } \quad \partial_{\mathbf{n}_{i}} \hat{M}^{n}=-f\left(S^{n-1}\right) \text { on } \Gamma_{\text {end }}^{n-1}, \\
\chi \hat{S}^{n}-d_{S} \Delta \hat{S}^{n}+\lambda \hat{S}^{n} & =k_{F} \hat{c}_{o x}^{n} \cdot \hat{M}^{n}-\gamma\left(\hat{c}_{o x}^{n}-c_{o x}^{t h}\right)_{+}+\chi S^{n-1} \circ\left(-\mathbf{v}^{n-1}\right),
\end{aligned}
$$

where, for instance, $c_{o x}^{n-1} \circ\left(-\mathbf{v}^{n-1}\right)$ stands for $c_{o x}^{n-1}\left(\mathbf{X}\left(t_{n-1} ; \cdot-\mathbf{v}^{n-1}\right)\right)$.

\subsection{Growth velocity}

The growth velocity $\hat{\mathbf{v}}^{n}$ on $\Omega_{i}^{n-1}$ is obtained by solving

$$
\begin{aligned}
-\operatorname{div} \mathbf{D}\left(\hat{\mathbf{v}}^{\mathrm{n}}\right)+\nabla \hat{\mathrm{q}}^{\mathrm{n}} & =0, & \mathbf{x} \in \Omega_{i}^{n-1}, \\
\operatorname{div} \hat{\mathbf{v}}^{\mathrm{n}} & =\frac{k_{F}}{A} \hat{c}_{o x}^{n} \cdot \hat{M}^{n}, & \mathbf{x} \in \Omega_{i}^{n-1}, \\
\mathbf{D}\left(\hat{\mathbf{v}}^{n}\right) \mathbf{n}_{i}-\hat{q}^{n} \mathbf{n}_{i} & =0, & \mathbf{x} \text { on } \Gamma_{\text {end }}^{n-1}, \\
\hat{\mathbf{v}}^{n} & =0, & \mathbf{x} \text { on } \partial \Omega_{i}^{n-1} \backslash \Gamma_{\text {end }}^{n-1} .
\end{aligned}
$$

Next, it is extended in an artificial velocity $\hat{\mathbf{v}}_{l}^{n}$ on $\Omega_{l}^{n-1}$ obtained as the solution of a Laplace problem with boundary condition $\hat{\mathbf{v}}_{l}^{n}=\hat{\mathbf{v}}_{i}^{n}$ on $\Gamma_{\text {end }}^{n-1}$ and homogeneous Dirichlet boundary conditions otherwise. Using this velocity we can move $\Omega_{i}^{n-1}$ and $\Omega_{l}^{n-1}$ and we can update all functions obtained so far in new functions defined on $\Omega_{i}^{n}$ and $\Omega_{l}^{n}$ by means of the FreeFem ++ interpolation method. Let us mention that because of the extreme smallness of the computed value $\hat{\mathbf{v}}^{n}$, which is inherited from the physiological phenomenon, we actually move the meshes with an artificial velocity $\kappa \hat{\mathbf{v}}^{n}, \kappa \gg 1$, in order to see significant changes in the geometry. We nevertheless take care of keeping the growth velocity in a range that prevents numerical diffusion. The loop $n-1$ to $n$ is now completed.

\section{Numerical Simulations}

\section{Physiological data}

We transform our model into a 2D model by an axisymmetry hypothesis. Homogeneous Neumann boundary conditions are classically imposed on the new boundary in order to enforce transverse continuity of the unknowns. Therefore, we work on a rectangular domain whose width is $l=0.5 \mathrm{~cm}$, that is a standard artery radius, and length is $L=5 \mathrm{~cm}$.

Let us now detail the parameter values. Some of them correspond to physiological data or boundary data that can be found in the literature, see [24], while others, namely those of the inflammatory model, cannot. We had to assign values to $k_{F}, r_{o x}, d_{o x}, d_{M}, d_{S}, \lambda$ and $\gamma$ in an artificial way. Further work should actually be devoted to parameter estimation. Recall that the entering velocity is a Poiseuille profile independent of time, we denote its maximal value by $U_{l, \max }$. As for the entering LDL concentration $C_{l, i n}$, it is supposed to be independent of space and time. Values are summarized in the tables below. 


\begin{tabular}{|l|c|c|c|}
\hline Lumen & $\rho=1.05 \mathrm{~g} \cdot \mathrm{cm}^{-3}$ & $\nu=0.035 \mathrm{~g} \cdot \mathrm{cm}^{-1} \cdot \mathrm{s}^{-1}$ & $D_{l}=2.867 \times 10^{-7} \mathrm{~cm}^{2} \cdot \mathrm{s}^{-1}$ \\
\hline Intima & $K=8.7 \times 10^{-13} \mathrm{~cm}^{2}$ & $\mu=0.03655 \mathrm{~g} \cdot \mathrm{cm}^{-1} \cdot \mathrm{s}^{-1}$ & $D_{i}=1.2 \times 10^{-7} \mathrm{~cm}^{2} \cdot \mathrm{s}^{-1}$ \\
\hline
\end{tabular}

\begin{tabular}{|l|l|l|l|}
\hline Lumen & $U_{l, \max }=24 \mathrm{~cm} \cdot \mathrm{s}^{-1}$ & $p_{\text {out }}=870 \mathrm{mmHg}$ & $C_{l, \text { in }}=3.12 \times 10^{-6} \mathrm{~g} \cdot \mathrm{cm}^{-3}$ \\
\hline Intima & & $p_{\text {med }}=800 \mathrm{mmHg}$ & \\
\hline
\end{tabular}

\begin{tabular}{|c|c|c|c|c|}
\hline Inflammation & $d_{o x}=10^{-3} \mathrm{~cm}^{2} \cdot \mathrm{s}^{-1}$ & $d_{M}=10^{-5} \mathrm{~cm}^{2} \cdot \mathrm{s}^{-1}$ & $d_{S}=10^{-3} \mathrm{~cm}^{2} \cdot \mathrm{s}^{-1}$ & \\
\hline & $k_{F}=1 \mathrm{~g}^{-1} \cdot \mathrm{cm}^{3} \cdot \mathrm{s}^{-1}$ & $r_{o x}=0.5$ & $\lambda=10 \mathrm{~s}^{-1}$ & $\gamma=1 \mathrm{~s}^{-1}$ \\
\hline
\end{tabular}

We finally turn to the Kedem-Katchalsky equations. From [24], we take $L_{p}=3 \cdot 10^{-11} \mathrm{~cm}^{3} \cdot d y n e^{-1} \cdot \mathrm{s}^{-1}$. As already mentioned, biomechanical properties of the endothelium are still a matter of active experimental research, see [14], [23], [18] among others, and a universal model for the endothelium permeability is yet to be obtained. We chose in (2.9) a wall shear stress-permeability relationship expected to assign a maximum value to areas with zero shear stress. We particularize (2.9) by considering that a standard permeability (perstand) corresponds to a standard value (wsstand) of the wall shear stress norm that we compute from a steady Poiseuille flow with $U_{l, \max }$ given above. More particularly,

$$
\xi(\boldsymbol{\sigma})=\frac{\text { perstand }}{\log 2} \times \log \left(1+\frac{2 \times \text { wsstand }}{|\boldsymbol{\sigma}|+\text { wsstand }}\right),
$$

with wsstand $=4 \nu \frac{U_{l, \max }}{l}$ and perstand $:=1.07 \times 10^{-11} \mathrm{~cm} \cdot \mathrm{s}^{-1}$.

\section{Model validation}

We compare our results with experiments performed in $[6,7]$ on mouse carotid arteries. In these experiments, the carotid geometry is modified by a perivascular cast that induces regions of lowered, increased, and lowered/oscillatory shear stresses. Mice are fed with a rich cholesterol diet. After some weeks, plaque formation together with plaque composition are studied. It is observed that atheromatous plaques develop in areas with lowered wall shear stress, namely right upstream of the cast and downstream of the cast. In addition, the plaque composition turns out to depend upon the wall shear stress pattern: plaques associated with low wall shear stress and laminar flow contain more oxidized LDL, whereas plaques located in zones of recirculating flow contain less oxidized LDL. We aim at obtaining numerical results based on our model that fit qualitatively with the experiments in terms of plaque location.

Figure 2 shows the initial two-dimensional geometry. The endothelium geometry of $[6,7]$ was reproduced by using Bézier interpolation. Initial finite element meshes contain 5312 triangles for the lumen and 3062 triangles for the intima. Figure 3 shows the time evolution of the wall shear stress tangential component. Figure 4 represents the lumen after 1500 time steps. As expected, two plaques emerged: the first one upstream the stenosed region, the second one downstream.

\section{Conclusions And Perspectives}

In the present work, we have extended the model that we previously proposed in [5] and that was restricted to reaction-diffusion equations for the biochemical species in the intima. We have coupled this inflammatory process with mass transfer from the lumen, in a so-called fluid-wall model, thus using fluid dynamics equations for the blood, transport equations for native LDL both in the lumen and in the intima, and transfer equations across the endothelium. Endothelium areas where the wall shear stress is low have been supposed to possess a higher permeability to native LDL. As in [5], the atheromatous plaque growth is driven by a velocity derived from an incompressibility assumption in the intima. We have performed computations in a two-dimensional 


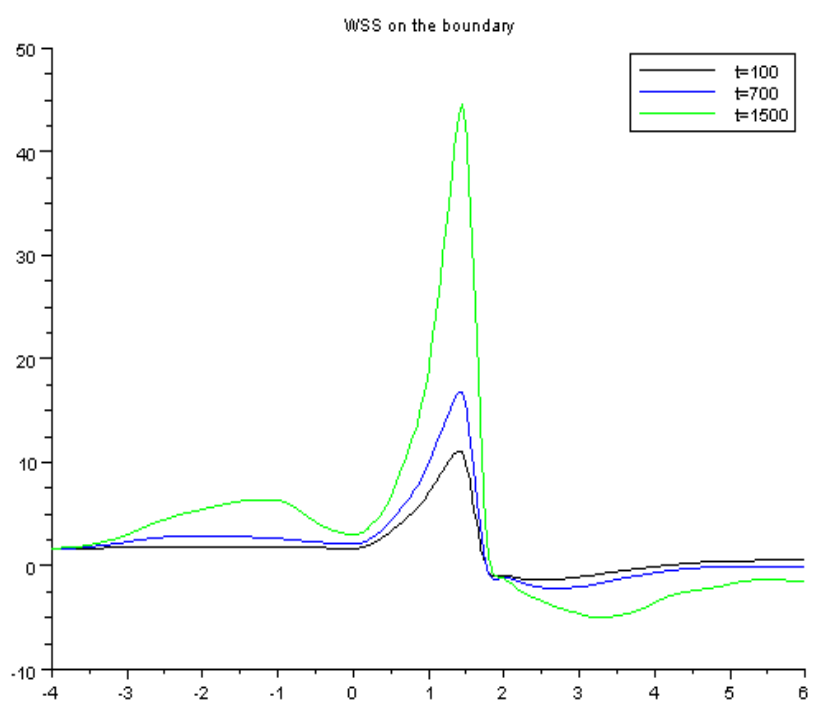

FiguRE 3. Time evolution of $\sigma \cdot \tau$ during simulation. The horizontal coordinate corresponds to a parametrisation of the endothelium with range parameter from -4 to 6 . The initially stenosed region is situated at the parameter value 1 .

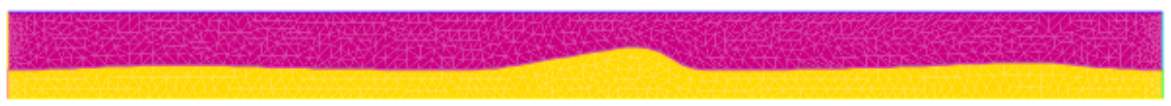

FiguRE 4. Lumen shape at the end of simulation, iteration $n=1500$.

geometry resulting from an axisymmetric configuration that mimicks Cheng et al. experiment and we have been able to numerically reproduce the emergence of plaques that is experimentally observed upst! ream and downstream of a perivascular cast. This is in good agreement with the clinical hypothesis that correlates atherosclerosis occurence with low wall shear stress. However, contrary to experiments, some thickening of the intima is numerically observed in the artificially stenosed region where the wall shear stress is high. This could be linked either to numerical diffusion or to a larger than expected species diffusion. Further work should be devoted to investigate the relative parts of the model and of its numerical approximation on this point.

More important, plaque formation is actually quite slow and it is not clear that taking a fully time-dependent model for blood was necessary. We will test simplified models for blood circulation that should circumvent many of the numerical possible artefacts and make the whole model analysis easier. The identification of the physiological parameters remains a major point. Several of them are out of experimental reach and parameter estimation techniques should be used. Finally, the mechanical behavior of the intima whose structure has been over-simplified and the endothelium response to shear stress could be improved. 


\section{REFERENCES}

[1] D. Ambrosi and L. Preziosi, On the closure of mass balance models for tumour growth, Math. Models Methods Appl. Sci. 12:737-754, 2002.

[2] D. Bresch, T. Colin, E. Grenier, B. Ribba and O. Saut, Computational modeling of solid tumor growth: the avascular stage, to appear in SIAM Jal of Numerical Analysis.

[3] D. Bresch, T. Colin, E. Grenier, B. Ribba and O. Saut, A viscoelastic model for avascular tumor growth, submitted.

[4] H.M. Byrne and L. Preziosi, Modelling solid tumour growth using the theory of mixtures, Math. Med. Biol. 20:341-366, 2004.

[5] V. Calvez, A. Ebde, N. Meunier and A. Raoult, Mathematical modeling of the atherosclerotic plaque formation, ESAIM Proceedings, 1-12, 2009.

[6] C. Cheng et al., Shear stress affects the intra- cellular distribution of eNOS: direct demonstration by a novel in vivo technique, Blood, 106, 3691-3698, 2005.

[7] C. Cheng et al., Atherosclerotic Lesion Size and Vulnerability Are Determined by Patterns of Fluid Shear Stress, Circ., 113, 2744-2753, 2006.

[8] A. Decoene, Modèle hydrostatique pour les écoulements à surface libre tridimensionnels et schémas numériques, Phd thesis, 2006.

[9] N. El Khatib, S. Genieys and V. Volpert, Atherosclerosis initiation modeled as an inflammatory process, Math. Model. Nat. Phenom. (2), 126-141, 2007.

[10] N. El Khatib, S. Genieys, B. Kazmierczak and V. Volpert, Reaction-Diffusion Model of Atherosclerosis Development, to appear.

[11] O. Kedem and A. Katchalsky, Thermodynamic analysis of the permeability of biological membranes to non-electrolytes, Biochim. Biophys. Acta 27, pp. 229-246, 1958.

[12] O. Kedem and A. Katchalsky, A physical interpretation of the phenomenological coefficients of membrane permeability, J. Gen. Physiol. 45, pp. 143-179, 1961.

[13] P. LibBy, Inflammation in atherosclerosis, Nature, 868-874, 2002.

[14] Z. Malat and A. Tedgui, Anti-inflammatory mechanisms in the vascular wall. Circ Res, 88, 877-887, 2001.

[15] G. Meyer, R. Merval and A. Tedgui, Effects of pressure-induced wall stretching and convection on low density lipoprotein and albumin uptake in the rabbit aorta, Circ Res, 79, 532-540, 1996.

[16] B. Maury, Characteristics ALE Method for the 3D Navier-Stokes Equations with a Free Surface, Int. Journal of Comp. Fluid Dyn. 6, 175-188, 1996.

[17] B. Osterud and E. Bjorklid, Role of monocytes in atherogenesis, Physiol Rev. 83: 1070-1086, 2003.

[18] G. Rappitsch and K. Perktold, Pulsatile albumin transport in large arteries: a numerical simulation study, ASME J. Biomech. Eng. 118, 511-519, 1996.

[19] M. Prosi, P. Zunino, K. Perktold and A. Quarteroni, Mathematical and numerical models for transfer of low-density lipoproteins through the arterial walls: a new methodology for the model set up with applications to the study of disturbed lumenal flow, J Biomech., 38: 903-917, 2005.

[20] A. Quarteroni and A. Valli, Domain Decomposition Methods for Partial Differential Equations, Oxford University Press, 1999.

[21] A. Quarteroni, A. Veneziani and P. Zunino, Mathematical and Numerical Modeling of the Solute Dynamics in Blood Flow and Arterial Walls, SIAM Jal of Numerical Analysis, 39, 1488-1511, 2002.

[22] R. Ross, Atherosclerosis - an inflammatory disease, Massachussets Medical Society 340: 115-120, 1999.

[23] J.M. TArbell, Mass transport in arteries and the localization of atherosclerosis, Annu. Rev. Biomed. Eng. 5: 79-118, 2003.

[24] P. Zunino, Mathematical and numerical modeling of mass transfer in the vascular system, Phd thesis, 2002. 